\title{
A Simple and Clean Synthesis of Polysubstituted 2,6-Dicyanoanilines Catalyzed by KF/alumina
}

\author{
Shubha Jain, Balwant S. Keshwal*, Deepika Rajguru, and Vasant W. Bhagwat \\ School of Studies in Chemistry, Vikram University, Ujjain, Madhya Pradesh 456010, India. \\ ${ }^{*}$ E-mail: bskeshwal2@gmail.com
}

(Received May 7, 2012; Accepted September 23, 2012)

\begin{abstract}
A simple and clean synthesis of polysubstituted 2,6-dicyanoanilines has been developed via the reaction of arylidenemalonodinitriles with 1-arylethylidenemalonodinitriles in ethanol catalyzed by KF/ alumina. Use of non-hazardous solid base as a catalyst, operational simplicity and improved product yields are the key advantages of the present protocol.
\end{abstract}

Key words: Polysubstituted 2,6-dicyanoanilines, Heterogeneous catalyst, Synthesis

\section{INTRODUCTION}

2,6-Dicyanoanilines are typical acceptor-donor-acceptor (A-D-A) systems comprising one electron-donor and two electron-acceptors. ${ }^{1}$ These molecular systems have attracted much attention because of their optical properties. ${ }^{2}$ They are the basis for artificial photosynthetic systems, ${ }^{3}$ materials presenting semi-conducting or nonlinear optical properties ${ }^{4}$ and molecular electronic devices. ${ }^{5}$ Moreover, 2,6-dicyanoanilines are useful intermediates for building blocks for cyclophanes to create a large molecular cavity and host-guest complex. ${ }^{6}$ In addition, their biaryl unit is represented in several types of compounds of current interest including natural products, polymers, liquid crystals, ligands and medicinal compounds. ${ }^{7}$ Also, these compounds exhibit strong fluorescence in UV light ${ }^{8}$ and may have utility as fluorescent materials. The amino and cyano groups can be converted into various other functional groups, hence these 2,6-dicyanoanilines can be used as starting materials for a number of aromatic compounds.

2,6-Dicyanoanilines have been reported to be prepared from arylidene-malonodinitriles and 1-arylethylidene-malonodinitriles in the presence of piperidine. ${ }^{9}$ Elgmeie et al. have synthesized these compounds by the reaction of cycloalkylidene-malonodinitriles with arylmethylene-cyanoacetamides catalyzed by $\mathrm{Et}_{3} \mathrm{~N} .{ }^{10}$ The reaction of chalcones with malononitrile also gave 2,6-dicyanoanilines. ${ }^{11}$ Further, they have been prepared under microwave irradiation $^{8}$ and using grinding method. ${ }^{12}$ However, most of these methods suffer from serious limitations, such as use of hazardous liquid base catalysts, unsatisfactory yields, tedious work-up and long reaction times. Therefore, the development of facile, efficient and eco-safe methods for their preparation is still necessary.

Organic reactions catalyzed by a solid heterogeneous catalyst have attracted much attention and offer advantages of operational simplicity, high selectivity and easy separation and recovery of the products, catalyst and solvent. Potassium fluoride coated alumina (KF/ alumina) has been recognized as an effective solid base catalyst to promote a wide range of organic reactions. ${ }^{13}$ In view of the above mentioned observations, we decided to investigate the catalytic activity of $\mathrm{KF}$ / alumina, an inexpensive, safe to handle and commercially available heterogeneous base, for the synthesis of polysubstituted 2,6-dicyanoanilines.

\section{EXPERIMENTAL}

All chemicals were purchased from Merck and SigmaAldrich as 'synthesis grade' and used without further purification. The key intermediates arylidene- and 1-arylethylidenemalonodinitriles were prepared via Knoevenagel reaction of the corresponding aldehyde or ketone with malononitrile as reported in the literature. ${ }^{16}$ Melting points were determined in open glass capillaries and are uncorrected. UV-vis absorption spectra were recorded on a Perkin Elmer Lambda 9 spectrometer. Fluorescence spectra were measured using a SPEX Fluorolog 3 spectrometer. IR spectra were recorded on a Perkin Elmer1430 spectrophotometer using potassium bromide pellets. ${ }^{1} \mathrm{H}$ and ${ }^{13} \mathrm{C}$ NMR spectra were obtained at $400 \mathrm{MHz}$ with a Bruker (AVANCE) spectrometer using DMSO-d $\mathrm{d}_{6}$ as solvent and TMS as an internal standard. MS spectra were measured at Micromass ZMD ESI $(70 \mathrm{eV})$ system. Elemental analyses were performed using Carlo Erba1108 analyzer. 


\section{General Procedure for the Synthesis of Polysubsti- tuted 2,6-Dicyanoanilines 3}

To a magnetically stirred solution of 1-arylethylidenemalonodinitrile 1 ( $1 \mathrm{mmol})$ and arylidenemalonodinitrile $2(1 \mathrm{mmol})$ in ethanol $(10 \mathrm{ml})$ was added $\mathrm{KF} /$ alumina $(10$ mol\%). The reaction mixture was stirred under reflux for 60-90 minutes to complete the reaction (monitored by TLC) and then allowed to cool at room temperature. The resulting precipitate was filtered and washed with ethanol $(25 \mathrm{ml})$ to obtain the pure product 3 .

\section{Characterization Data for the Representative Com- pounds}

3-Amino-5-(furan-2-yl)-4'-nitro-biphenyl-2,4-dicarbonitrile (3g): Yellow solid; Yield 74\%; m.p. $277-278^{\circ} \mathrm{C}$; Anal. Calcd. for $\mathrm{C}_{18} \mathrm{H}_{10} \mathrm{~N}_{4} \mathrm{O}_{3}: \mathrm{C}, 65.45 ; \mathrm{H}, 3.05 ; \mathrm{N}, 16.96 \%$. Found: C, 65.76; H, 3.27; N, 16.72\%; UV-vis $\left(\mathrm{CH}_{2} \mathrm{Cl}_{2}\right.$, $\left.\lambda_{\max } / \mathrm{nm}\right): 363$; IR $\left(\mathrm{KBr}, \mathrm{cm}^{-1}\right): 3472,3365,2925,2212$, 1637, 1540, 1296, 1039, 824; ${ }^{1} \mathrm{H}$ NMR (400 MHz, DMSO-d 6 ,

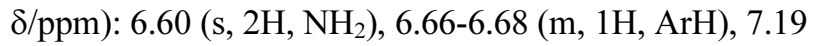
(s, 1H, ArH), 7.44 (d, 1H, ArH, J=3.5 Hz), 7.77 (d, 1H, ArH, $J=1.7 \mathrm{~Hz}$ ), 7.82-7.84 (m, 2H, ArH), 8.36-8.38 (m, $2 \mathrm{H}, \mathrm{ArH}) ;{ }^{13} \mathrm{C}$ NMR (400 MHz, DMSO-d 6 , $\left.\delta / \mathrm{ppm}\right): 89.96$, 93.01, 99.49, 112.50, 112.90, 114.01, 115.32, 115.78, 123.52, 129.52, 136.48, 143.68, 144.80, 147.21, 147.77, 148.41, 154.17; MS: $m / z=331\left(\mathrm{M}^{+}\right)$.

3-Amino-4'-nitro-5-(thiophen-2-yl)biphenyl-2,4-dicarbonitrile (3h): Yellow solid; Yield $68 \%$; m.p. $272-273{ }^{\circ} \mathrm{C}$; Anal. Calcd. for $\mathrm{C}_{18} \mathrm{H}_{10} \mathrm{~N}_{4} \mathrm{O}_{2} \mathrm{~S}: \mathrm{C}, 62.42 ; \mathrm{H}, 2.91 ; \mathrm{N}$, 16.18\%. Found: C, 62.68; H, 2.96; N, 16.34\%; UV-vis $\left(\mathrm{CH}_{2} \mathrm{Cl}_{2}, \lambda_{\max } / \mathrm{nm}\right): 358 ; \mathrm{IR}\left(\mathrm{KBr}, \mathrm{cm}^{-1}\right): 3458,3260$, 3131, 3088, 2293, 1649, 1555, 1342, 1053, 790; ${ }^{1} \mathrm{H}$ NMR (400 MHz, DMSO-d 6 , $\delta / \mathrm{ppm}): 6.74$ (s, 2H, NH $\left.{ }_{2}\right), 6.91$ (s, 1H, ArH), 7.22 (t, 1H, ArH, $J=4.6 \mathrm{~Hz}), 7.70-7.73$ (m, 2H, ArH), 7.84 (d, 2H, ArH, $J=8.6 \mathrm{~Hz}), 8.36$ (d, 2H, ArH, $J=$ $8.6 \mathrm{~Hz}$ ); ${ }^{13} \mathrm{C}$ NMR $(400 \mathrm{MHz}$, DMSO-d 6 , $8 / \mathrm{ppm}): 96.73$, 96.77, 115.00, 115.29, 117.52, 123.54, 128.06, 128.82, 129.68, 133.24, 138.04, 141.75, 143.45, 147.34, 152.28, 154.31; MS: $m / z=347\left(\mathrm{M}^{+}\right)$.

2-Amino-4-(furan-2-yl)-6-(thiophen-2-yl)isophthalonitrile (3i): Yellow rods; m.p. 323-325 ${ }^{\circ} \mathrm{C}$; Anal. Calcd. for $\mathrm{C}_{16} \mathrm{H}_{9} \mathrm{~N}_{3} \mathrm{OS}$ : C, $65.96 ; \mathrm{H}, 3.11 ; \mathrm{N}, 14.42 \%$. Found: $\mathrm{C}$, 65.98; H, 3.24; N, 14.37\%; UV-vis $\left(\mathrm{CH}_{2} \mathrm{Cl}_{2}, \lambda_{\max } / \mathrm{nm}\right)$ : 368; IR $\left(\mathrm{KBr}, \mathrm{cm}^{-1}\right): 3452,3281,2224,1590,1570,1357$, $1235,1148,741 ;{ }^{1} \mathrm{H}$ NMR $(400 \mathrm{MHz}$, DMSO-d 6 , $\delta / \mathrm{ppm})$ : $6.31\left(\mathrm{~s}, 2 \mathrm{H}, \mathrm{NH}_{2}\right), 6.65-6.66(\mathrm{~m}, 2 \mathrm{H}, \mathrm{ArH}), 7.41-7.42(\mathrm{~m}$, $2 \mathrm{H}, J=3.6 \mathrm{~Hz}, \mathrm{ArH}), 7.58$ (s, 1H, ArH), $7.74(\mathrm{~m}, 2 \mathrm{H}$,
ArH); ${ }^{13} \mathrm{C}$ NMR (400 MHz, DMSO-d 6 , $\left.\delta / \mathrm{ppm}\right): 88.70$, $91.60,112.52,112.72,113.37,116.04,116.07,128.05$, $128.49,128.87,136.26,138.38,141.44,144.94,148.41$, 154.68; MS: $m / z=292\left(\mathrm{M}^{+}\right)$.

2-Amino-4,6-di(thiophen-2-yl)isophthalonitrile (3j): Yellow rods; m.p. $314-316^{\circ} \mathrm{C}$; Anal. Calcd. for $\mathrm{C}_{16} \mathrm{H}_{9} \mathrm{~N}_{3} \mathrm{~S}_{2}$ : C, 62.52; H, 2.95; N, 13.67\%. Found: C, 62.71; H, 2.82, $\mathrm{N}, 13.56 \%$; UV-vis $\left(\mathrm{CH}_{2} \mathrm{Cl}_{2}, \lambda_{\max } / \mathrm{nm}\right): 372 ; \mathrm{IR}\left(\mathrm{KBr}, \mathrm{cm}^{-1}\right)$ : 3376, 3195, 2223, 1662, 1576, 1336, 1262, 835, 751; ${ }^{1} \mathrm{H}$

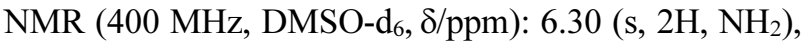
6.69-6.71 (m, 2H, ArH), 7.41-7.42 (m, 2H, ArH), 7.53 (s, $1 \mathrm{H}, \mathrm{ArH}), 7.83$ (m, 2H, ArH) ${ }^{13} \mathrm{C}$ NMR (400 MHz, DMSO$\left.\mathrm{d}_{6}, \delta / \mathrm{ppm}\right): 92.14,115.96,117.04,128.06,128.59,128.95$, 138.12, 141.47, 154.70; MS: $m / z=308\left(\mathrm{M}^{+}\right)$.

2-Amino-4-(furan-2-yl)-6-(5-methyl-thiophen-2-yl)isophthalonitrile (3k): Yellow needles; m.p. $334-335^{\circ} \mathrm{C}$; Anal. Calcd. for $\mathrm{C}_{17} \mathrm{H}_{11} \mathrm{~N}_{3} \mathrm{OS}$ : C, 66.87; H, 3.63, N, $13.76 \%$. Found: C, 66.94; H, 3.48, N, $13.58 \%$; UV-vis $\left(\mathrm{CH}_{2} \mathrm{Cl}_{2}\right.$, $\left.\lambda_{\max } / \mathrm{nm}\right): 365$; IR $\left(\mathrm{KBr}, \mathrm{cm}^{-1}\right): 3458,3260,3088,2293$, 1649, 1555, 1342, 1253, 1091, 790; ${ }^{1} \mathrm{H}$ NMR $(400 \mathrm{MHz}$, DMSO-d $\left._{6}, \delta / \mathrm{ppm}\right): 2.12\left(\mathrm{~s}, 3 \mathrm{H}, \mathrm{CH}_{3}\right), 6.30\left(\mathrm{~s}, 2 \mathrm{H}, \mathrm{NH}_{2}\right)$, $6.98(\mathrm{~s}, 1 \mathrm{H}, \mathrm{ArH}), 7.20-7.22(\mathrm{~m}, 1 \mathrm{H}, \mathrm{ArH}), 7.65(\mathrm{~d}, 1 \mathrm{H}$, $J=0.9 \mathrm{~Hz}, \mathrm{ArH}), 7.67$ (d, $1 \mathrm{H}, J=0.9 \mathrm{~Hz}, \mathrm{ArH}), 7.69$ (d, $1 \mathrm{H}, J=1.0 \mathrm{~Hz}, \mathrm{ArH}), 7.70(\mathrm{~d}, 1 \mathrm{H}, J=1 \mathrm{~Hz}, \mathrm{ArH}) ;{ }^{13} \mathrm{C} \mathrm{NMR}$ (400 MHz, DMSO-d 6 , $\delta / \mathrm{ppm}): 14.15,92.04,108.94,110.28$, $111.41,117.88,118.65,122.55,123.73,127.43,135.74,136.96$, 145.82, 146.39, 150.48, 154.60, 163.30; MS: $m / z=306\left(\mathrm{M}^{+}\right)$.

\section{RESULTS AND DISCUSSION}

As part of our current studies on developing clean synthesis methodologies, we describe herein a convenient and efficient procedure for the synthesis of polysubstituted 2,6-dicyanoanilines by the reaction of 1-arylethylidenemalonodinitriles with arylidenemalonodinitriles using $\mathrm{KF} /$ alumina as a strongly basic heterogeneous catalyst and thus, avoiding use of hazardous liquid organic bases like piperidine and triethylamine.

In order to optimize the reaction conditions, the reaction of 2-(1-phenylethylidine)malononitrile 1a and 2-(4nitrobenzylidene)malononitrile $\mathbf{2 a}$ as a model reaction was carried out in ethanol under reflux in presence of different catalysts, for example, $\mathrm{ZnO}$, basic alumina, $\mathrm{KF}, \mathrm{K}_{2} \mathrm{CO}_{3}$ and $\mathrm{KF} /$ alumina to afford 3a. The results obtained with different catalysts are summarized in Table 1.

The best results in terms of reaction, time and yield were obtained with a catalytic amount of $\mathrm{KF} /$ alumina under 
Table 1. Synthesis of 3a in ethanol in presence of different catalysts under reflux

\begin{tabular}{cccc}
\hline Entry & Catalyst (mol\%) & Time (h) & Yield $^{\mathrm{a}}(\%)$ \\
\hline 1 & $\mathrm{ZnO}(10)$ & 6 & 24 \\
2 & Basic alumina (10) & 6 & 19 \\
3 & $\mathrm{KF}(10)$ & 4 & 35 \\
4 & $\mathrm{~K}_{2} \mathrm{CO}_{3}(10)$ & 6 & $\mathrm{Nil}$ \\
5 & $\mathrm{KF} /$ alumina $(10)$ & 1 & 72 \\
$6^{\mathrm{b}}$ & $\mathrm{KF} /$ alumina $(10)$ & 8 & 38 \\
\hline
\end{tabular}

${ }^{\text {a Isolated yield. }}$

${ }^{\mathrm{b}}$ The reaction was carried out at room temperature.

reflux. The product formation was also observed when the reaction was carried out at room temperature in ethanol in the presence of $\mathrm{KF} /$ alumina $(10 \mathrm{~mol} \%)$. The reaction, however, required longer reaction time and the product yield was inferior (38\%, Table 1, entry 6).

Under the optimized reaction conditions, various kinds of 1-arylethylidenemalonodinitriles $\mathbf{1}$ and arylidenemalonodinitriles 2 were made to react to give the corresponding polysubstituted 2,6-dicyanoaniline derivatives 3 (Table 2). As can be seen from Table 2 , all of $\mathbf{1}$ and $\mathbf{2}$, either bearing electron-withdrawing or electron-donating groups, afforded the desired products $\mathbf{3}$ in high to excellent yields under same reaction conditions. It is noteworthy that the methodology worked well for hetroaromatic substrates (Table 2, entries 7-11).

Products 3d and 3e, however, were obtained in slightly poor yields (Table 2, entries 4 and 5). These results were found to be consistent with previous reports. ${ }^{9}$

The known compounds were characterized by ${ }^{1} \mathrm{H}$ NMR spectra and comparing their melting points with those reported in the literature. The structures of other products were elucidated by UV, IR, ${ }^{1} \mathrm{H}$ NMR, ${ }^{13} \mathrm{C}$ NMR, Mass spectral studies and elemental analysis. The IR spectrum of $\mathbf{3 i}$, for instance, exhibited the absorption bands at 3452 and $3281 \mathrm{~cm}^{-1}$ due to the presence of $\mathrm{N}-\mathrm{H}$ (amine). The bands at $2224 \mathrm{~cm}^{-1}$ and $1570 \mathrm{~cm}^{-1}$ depicted the presence of $\mathrm{CN}$ (nitrile) and $\mathrm{C}=\mathrm{C}$ (aromatic) respectively. The ${ }^{1} \mathrm{H}$ NMR spectrum of $3 \mathbf{i}$ in DMSO- $\mathrm{d}_{6}$ demonstrated a characteristic singlet at $\delta 6.31$ for the aromatic $\mathrm{NH}_{2}$ and different signals from $\delta 6.65$ to $\delta 7.74$ for the aromatic protons. The mass spectrum of $3 \mathbf{i}$ showed the molecular ion peak at $\mathrm{m} / \mathrm{z}$ 292 corresponding to its molecular weight.

The compound 3i strongly absorbs in the violet as evidenced by its yellow colour. The UV-visible spectrum of $3 \mathbf{i}$ recorded in dichloromethane showed a strong absorption band at $368 \mathrm{~nm}$ while upon UV excitation, $3 \mathbf{3}$ showed an intense blue fluorescence with maximum emission at $407 \mathrm{~nm}$ and a fluorescence quantum yield of $\Phi_{\mathrm{f}}=0.10$ (using 9,10-diphenylanthracene as standard). ${ }^{14}$ The observed photophysical properties and high fluorescence quantum yield are identical to those previously found for 2,6-dicyanoanilines. ${ }^{15}$

In accordance with the mechanism suggested in literature, ${ }^{9}$ the first step of the present reaction may involve the vinylogous Michael addition of $\mathbf{1}$ to arylidenemalonodinitriles $\mathbf{2}$ to give adduct $\mathbf{A}$. The addition is followed by Thorpe cyclization of Michael product $\mathbf{A}$ to the cyclohexadiene system $\mathbf{B}$. Finally, the elimination of hydrogen cyanide from $\mathbf{B}$ gave the polysubstituted 2,6-dicyanoanilines (Scheme 1).

Table 2. KF/ alumina catalyzed synthesis of polysubstituted 2,6-dicyanoanilines

\begin{tabular}{|c|c|c|c|c|c|c|}
\hline Entry & $\mathrm{Ar}$ & Ar' & Product & Time (min) & Yield (\%) & m.p. $\left({ }^{\circ} \mathrm{C}\right)$ Found [reported] \\
\hline 1 & $\mathrm{C}_{6} \mathrm{H}_{5}$ & $4-\mathrm{O}_{2} \mathrm{NC}_{6} \mathrm{H}_{4}$ & $3 a$ & 60 & 72 & $242-245[244-246]^{11 d}$ \\
\hline 2 & $\mathrm{C}_{6} \mathrm{H}_{5}$ & $3-\mathrm{O}_{2} \mathrm{NC}_{6} \mathrm{H}_{4}$ & $3 b$ & 60 & 73 & $236-237[234-236]^{9}$ \\
\hline 3 & $4-\mathrm{O}_{2} \mathrm{NC}_{6} \mathrm{H}_{4}$ & $4-\mathrm{O}_{2} \mathrm{NC}_{6} \mathrm{H}_{4}$ & $3 \mathrm{c}$ & 90 & 62 & $349-351[352-353]^{9}$ \\
\hline 4 & $\mathrm{C}_{6} \mathrm{H}_{5}$ & $2-\mathrm{CH}_{3} \mathrm{OC}_{6} \mathrm{H}_{4}$ & $3 d$ & 80 & 45 & $167-170[168-169]^{11 d}$ \\
\hline 5 & $\mathrm{C}_{6} \mathrm{H}_{5}$ & $4-\mathrm{H}_{2} \mathrm{NC}_{6} \mathrm{H}_{4}$ & $3 \mathrm{e}$ & 90 & 42 & $320-323[321-322]^{9}$ \\
\hline 6 & $4-\mathrm{O}_{2} \mathrm{NC}_{6} \mathrm{H}_{4}$ & 4- $\mathrm{H}_{2} \mathrm{NC}_{6} \mathrm{H}_{4}$ & $3 \mathrm{f}$ & 70 & 64 & $287-290[286-287]^{9}$ \\
\hline 7 & $4-\mathrm{O}_{2} \mathrm{NC}_{6} \mathrm{H}_{4}$ & 2-Furanyl & $3 g$ & 60 & 74 & $277-278$ \\
\hline 8 & $4-\mathrm{O}_{2} \mathrm{NC}_{6} \mathrm{H}_{4}$ & 2-Thienyl & $3 h$ & 60 & 68 & $272-273$ \\
\hline 9 & 2-Thienyl & 2-Furanyl & $3 \mathrm{i}$ & 60 & 72 & $323-325$ \\
\hline 10 & 2-Thienyl & 2-Thienyl & $3 \mathrm{j}$ & 60 & 69 & $314-316$ \\
\hline 11 & 2-Furanyl & 5-Me-2-Thienyl & $3 \mathrm{k}$ & 70 & 60 & $334-335$ \\
\hline
\end{tabular}




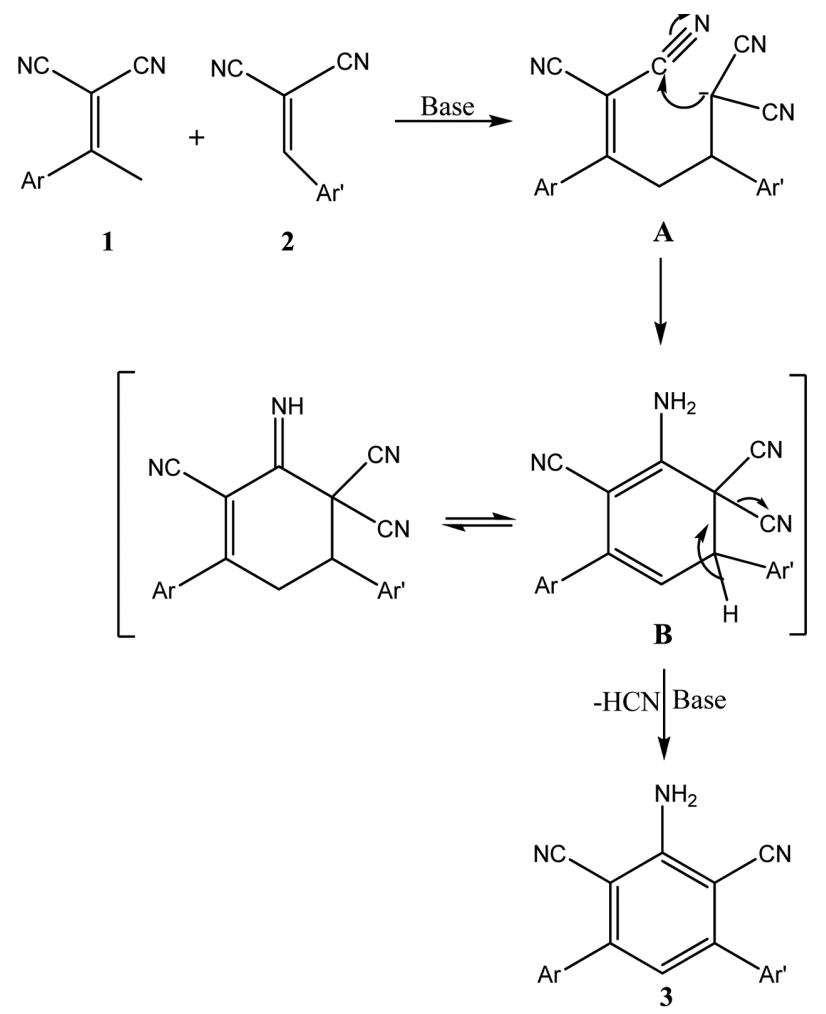

Scheme 1. Plausible mechanism for the formation of polysubstituted 2,6-dicyanoanilines $\mathbf{3}$.

\section{CONCLUSION}

In summary, a clean and efficient method for the synthesis of polysubstituted 2,6-dicyanoanilines has been developed via the reaction of 1-arylethylidenemalonodinitriles with arylidenemalonodinitriles in ethanol under reflux using $\mathrm{KF}$ / alumina as a heterogeneous green catalyst.

Acknowledgments. We thank The Director, SAIF, Punjab University, Chandigarh for NMR and MS spectral data. We are grateful to Dr. Aman Deep Acharya for his scientific suggestions.

\section{REFERENCES}

1. (a) Dumur, F.; Gautier, N.; Gallego-Planas, N.; Sahin, Y.; Levillain, E.; Mercier, N.; Hudhomme, P. J. Org. Chem. 2004, 69, 2164. (b) Depaemelaere, S.; De Schryver, F. C.; Verhoeven, J. W. J. Phys. Chem. A 1998, 102, 2109. (c) Xiao, Y.; Qian, X. H. Tetrahedron Lett. 2003, 44, 2087.

2. (a) Sepiol, J.; Milart, P. Tetrahedron 1985, 41, 5261.(b) Griffiths, J.; Lockwood, M.; Roozpeikar, B. J. Chem. Soc. Perkin Trans. 2 1977, 1608.

3. (a) Fox, M. A.; Chanon, M. Eds. Photoinduced Electron
Transfer 1988. (b) Kurreck, H.; Huber, M. Angew. Chem. Int. Ed. 1995, 34, 849.

4. (a) Prasad, P. N.; Williams, D. J. Introduction to Nonlinear Optical Effects in Molecules and Polymers; Wiley: NewYork, 1991. (b) Nalwa, H. S. Adv. Mater. 1993, 5, 341. (c) Long, N. J. Angew. Chem. Int. Ed. 1995, 34, 21. (d) Wong, M. S.; Bosshard, C.; Pan, F.; Gunter, P. Adv. Mater. 1996, 8, 677.

5. (a) Metzger, R. M.; Panetta, C. New. J. Chem. 1991, 15, 209. (b) Launay, J. P.; Ferry, D. K. Molecular Electronics In Granular Nanoelectronics; Plenum Press: New York, 1991. (c) Petty, M. C.; Bryce, M. R.; Bloor, D. Introduction to Molecular Electronics; Oxford University Press: New York, 1995.

6. Raghukumar, V.; Murugan, P.; Ramakrishnan, V. T. Synth. Commun. 2001, 31, 3497.

7. (a) Goel, A.; Singh, F. V. Tetrahedron Lett. 2005, 46, 5585 (b) Andersen, N. G.; Maddaford, S. P.; Keay, B. A. J. Org. Chem. 1996, 61, 9556 (c) Noyori, R. Chem. Soc. Rev. 1989, 18, 187 (d) Yamamura, K.; Ono, S.; Ogoshi, H.; Masuda, H. Y.; Kuroda, Y. Synlett 1989, 18; (e) Yamamura, K.; Ono, S.; Tabushi, I. Tetrahedron Lett. 1988, 29, 1797.

8. Cui, S. L.; Lin, X. F.; Gang, W. Y. J. Org. Chem. 2005, 70, 2866.

9. Milart, P.; Wilamowski, J.; Sepiol, J. J. Tetrahedron 1998, $54,15643$.

10. Elgemei, G. E. H.; Elzanate, A. M.; Mansour, A. K. J. Chem. Soc. Perkin Trans. 1 1992, 1, 1073.

11. (a) Victory, P. J.; Borrell, J. I.; Vidal-Ferran, A. Heterocycles 1993, 36, 769. (b) Victory, P.; Borrell, J. I.; VidalFerran, A.; Seoane, C.; Soto, J. Tetrahedron Lett. 1991, 32, 5375. (c) Victory, P. J.; Borrell, J.; Vidal-Ferran, A.; Montenegro, E.; Jimeno, M. L. Heterocycles 1993, 36, 2273. (d) Xin, X.; Wang, Y.; Xu, W.; Lin, Y.; Duan, H.; Dong, D. Green Chem. 2010, 12, 893.

12. Rong, L.; Han, H; Jiang H; Tu S. Synth. Commun. 2008 , 38,3530 .

13. (a) Yamawaki, J.; Ando, T. Chem. Lett. 1979, 755. (b) Clark, J. H. Chem. Rev. 1980, 80, 429. (c) Wang, X. S.; Zhang, M. M.; Li, Q.; Yao, C. S.; Tu, S. J. Synth. Commun. 2008, 38, 1896. (d) Blass, B. E. Tetrahedron 2002, 58, 9302. (e) Schmittling, E. A.; Sawyer, J. S. Tetrahedron Lett. 1991, 32, 7207. (f) Basu, B.; Das, P.; Das, S. Curr. Org. Chem. 2008, 12, 141.

14. (a) Lakowicz, J. R. Principles of Fluorescence Spectroscopy, 2nd ed.; Kluwer Academic/ Plenum Publishers: New York, 1999. (b) Demas, J. N.; Grosby, G. A. J. Phys. Chem. 1971, 75, 991.

15. Oshima, J.; Yoshihara, T.; Tobita, S. Chem. Phys. Lett. 2006, 423, 306.

16. (a) Xue, D.; Chen, Y. C.; Cui, X.; Wang, Q.W.; Zhu, J.; Deng, J. G. J. Org. Chem. 2005, 70, 3584 (b) Sandhu, Suresh; Jagir, S. Green Chem. Lett. and Rev. 2009, 2, 189 (c) Mukhopadhyay, C.; Datta, A. Synth. Commun. 2008, 38, 2103. 